\title{
PRISMA - Practical meta-analysis of applying local triamcinolone acetonide injection for stenosis after esophageal cancer surgery
}

This article was published in the following Dove Press journal:

Cancer Management and Research

\section{Wu Wang' \\ Zhen Shen² \\ Baoxin $\mathrm{Du}^{3}$ \\ Yanyang Pang ${ }^{4}$}

'Laboratory of Tropical Biomedicine and Biotechnology, School of Tropical Medicine and Laboratory Medicine, Hainan Medical University, Haikou, Hainan 570100, China; ${ }^{2}$ Division of Liver Disease, Huangshi City Hospital of Traditional Chinese Medicine (Infectious Disease Hospital), Edong Healthcare Group, Huangshi, Hubei 435000, China; ${ }^{3}$ Department of Urology, Chongqing Hospital of Traditional Chinese Medicine, Chongqing 402760, China; ${ }^{4}$ Department of Traditional Chinese Medicine, Hainan Medical University, Haikou, Hainan 570100, China
Correspondence: Yanyang Pang Department of Traditional Chinese Medicine, Hainan Medical University, No. 3 Xueyuan Road, Longhua District, Haikou City, Hainan Province 570100,

China

Tel +8689866890539

Email pangyanyang2009@163.com
Objectives: To explore the practical method of endoscopic triamcinolone acetonide (TA) injection immediately after endoscopic surgery and combined with endoscopic dilation (ED) in the management of stenosis after esophageal cancer surgery based on their efficacy and safety. Methods: A comprehensive search was performed in electronic databases including MEDLINE, EMBASE, the Cochrane Library, Web of Science, and China National Knowledge Infrastructure for possible controlled studies. Meta-analyses of the included studies were completed using Reviewer Manager software and were reported based on Preferred Reporting Items for Systematic Reviews and Meta-Analyses.

Results: Eight randomized studies and five controlled studies containing 575 patients were obtained. In five studies ( $\mathrm{n}=282$ ), TA injected after surgery reduced the risk of stenosis (risk difference $[\mathrm{RD}]=-0.51,95 \% \mathrm{CI}[-0.64,-0.39], P<0.01)$ and the required $\mathrm{ED}$ sessions $(\mathrm{RD}$ $=-3.66,95 \%$ CI $[-5.87,-1.46], P<0.01)$. In eight studies $(\mathrm{n}=293)$, TA injection combined with ED reduced the risk of recurrence of stenosis $(\mathrm{RD}=-0.28,95 \% \mathrm{CI}[-0.47,-0.08], P<0.01)$ and the required ED sessions $(\mathrm{RD}=-0.71,95 \% \mathrm{CI}[-1.39,-0.04], P<0.05)$. TA injection therapy did not increase the risk of complications in seven studies $(n=380 ; \mathrm{RD}=-0.01,95 \% \mathrm{CI}[-0.04$, 0.02], $P=0.53$ ) compared with control.

Conclusion: TA injection therapy after esophageal cancer surgery and combined with ED are both effective and safe in the management of stenosis, as they reduce the risk of stenosis and sequentially the required ED sessions without increasing complications.

Keywords: stenosis, esophageal cancer, surgery, triamcinolone acetonide, meta-analyses

\section{Introduction}

Esophageal stenosis are mainly caused by peptic injury, corrosive injury, endoscopic submucosal dissection (ESD), surgical anastomosis, and radiation therapy for esophageal cancer. ${ }^{1,2}$ Recently, incidence of surgery-related stenosis is increasing due to the widespread application of ESD, which has been the first choice for superficial lesions and early esophageal cancer. ${ }^{3,4}$ However, about $90 \%$ of patients with mucosal defects of circumference $>3 / 4$ and $40 \%$ of patients who had undergone esophagectomy will experience postoperative stenosis. ${ }^{5,6}$ Dysphagia discomfort and impaired quality of life persist in the patients' life due to stenosis formation during the process of edema, fibrotic tissue proliferation, and collagen accumulation. ${ }^{4}$

Although proton pump inhibitors have been developed and have played important roles in the management of specific stenosis after peptic injury, most of the other stenoses can be handled by additional serial endoscopic dilation (ED) irrespective of 
etiology. ${ }^{7}$ While ED often needs to be frequently repeated in some patients, more required numbers of ED are associated with higher risk of perforation. ${ }^{8}$ The intended management of esophageal stenosis was to improve quality of life of the patients with lower incidence of recurrence and complication.

Within the kinds of proposed therapies, local intralesional injection of corticosteroids is currently supposed to be promising, because of its pharmacological effects of inflammatory response inhibition and fibrotic tissue reduction, and also its low cost and clinical feasibility. ${ }^{9,10}$ Among the injected corticosteroids, triamcinolone acetonide (TA) was mostly adopted, as it demonstrated effectiveness in treating hypertrophic scars and keloids. ${ }^{11}$

However, the practical details of TA injection were not fully described, and to some extent confusing, and the roles of TA injection after esophageal cancer surgery for preventing stenosis occurrence and in combination with ED for preventing stenosis recurrence were not clearly elaborated. Available studies addressing this issue were limited, and the sample size of most of them was small. Therefore, a meta-analysis including all relevant studies was conducted to assess the clinical efficacy, safety, and practical details of TA injection for the management of esophagus stenosis.

\section{Methods}

This meta-analysis was conducted in accordance with the Cochrane Handbook for Systematic Reviews of Interventions $^{12}$ and was reported in accordance with the Preferred Reporting Items for Systematic Reviews and Meta-Analyses recommendations. ${ }^{13}$

\section{Search strategy}

Literature search was conducted through retrieving electronic databases including the Cochrane Library (March 2018 issue), MEDLINE (2000 to March 2018), EMBASE (2000 to March 2018), Web of Science (2000 to March 2018), and China National Knowledge Infrastructure (March 2018 issue). Search terms were as follows: (esophageal OR esophagus OR esophagus) AND (stenoses OR stricture OR stenosis) AND (TA OR triamcinolone acetonide). No language and time limits were applied. References of similar and referred case reports, comparative studies, and reviews were also scanned to manually search potential articles. Two reviewers independently reviewed the search results according to the inclusion criteria. Disagreements between reviewers were resolved in consultation with a third reviewer.

\section{Inclusion criteria}

1. Types of studies - Clinical prospective randomized controlled trials were targeted in the selection process, while observational studies were also considered to achieve an increase of overall sample size.

2. Types of participants - Patients submitted to endoscopic surgery of ESD, and open surgery involving transection and anastomosis for esophageal cancer.

3. Types of interventions - Studies comparing endoscopic TA injection with control. Two kinds of injection method were focused: after endoscopic surgery before stenosis occurrence and combined with ED after stenosis diagnosis.

4. Types of outcome measures - The main outcome measurement was stenosis rate. Secondary outcome measures were number of ED sessions, dysphagia-free interval, and treatment-related complications. Stenosis was defined after finding evidence of impossibility of passing by a normal endoscope. Dysphagia-free interval was defined as the time duration from surgery or dilation completion to a recurrent stenosis. Treatment-related complications included perforation, bleeding, mucosal tearing, and local infection, which were classified as severe (which can lead to death and required emergency procedures to control) and nonsevere (which can be effectively controlled by internal medicine).

\section{Data extraction}

Another two reviewers independently extracted the data including basic information, outcome measures, and methodological quality items by using a normal extraction method. Basic information included first author, publication year, case, age of patients, type of surgery, mucosal defect of circumference, information of TA injection procedure, TA dose and times, primary disease due to surgery, and followup periods. Combined data included main and secondary outcome measures. Any disagreements between the reviewers were resolved by discussion.

\section{Risk of bias of individual studies}

To verify methodological quality of eligible studies, two reviewers were working independently to assess the risk of bias in randomized controlled trials on randomization, allocation concealment, blinding, withdrawal and dropout, selective reporting result, and other bias, and to assess quality using the scale of Jadad score according to the recommendations in the Cochrane Handbook for Systematic Reviews of Interventions. ${ }^{12}$ For observational studies, the same reviewers 
used Newcastle-Ottawa Quality Assessment Scale for risk of bias assessment based on patient representativeness, cohort comparability, and outcome assessment. An included study required a scale score $>5$, demonstrating a low risk of bias. ${ }^{14}$

\section{Statistical analysis}

Review Manager 5.3 (from the website of the Cochrane Collaboration, Copenhagen, Denmark) was used to analyze data. Risk of bias across the individual studies was assessed by the chi-squared test, which indicated the relationship between sample size and effect size (statistical heterogeneity), and was presented as forest plots for each outcome measurement based on the combined model according to $I^{2}$. When $I^{2}>50 \%$, random-effect model was used, and if $I^{2} \leq 50 \%$, the fixed-effect model was used. Using Mantel-Haenszel test, risk difference (RD) with 95\% CI was calculated for combined effect size of dichotomous variables, and standard mean difference (SMD) or mean difference (MD) was calculated for combined effect size of continuous variables. $P<0.05$ was considered of statistical significance.

Additional analysis included the following:

1. Subgroup analysis - Types of study design (random or not), TA injection method (after surgery or combined with ED), different injection times (one time or two or more times), TA dose ( $\leq 50 \mathrm{mg},>50 \mathrm{mg}$, or specific dose), and TA injection timing when combined with ED (before ED or after ED) were separately evaluated as key factors in clinical practice. Through comparing the difference between subgroups, the reliability and clinical application would be primarily judged for medical practice on specific patient population.

2. Publication bias - Inverted funnel plot was visually judged for each outcome measure.

\section{Results}

\section{Summary of the included studies}

We retrieved 587 references from electronic databases and other resources, and obtained 14 full-text articles after reading abstracts according to inclusion criteria. Finally, 13 studies including 280 patients in steroid group and 295 patients in control group were included for meta-analysis, as shown in Figure 1. ${ }^{15-27}$ There were eight randomized trials (six blank control with no additional injection and two sham control with saline injection) and five observational studies with no additional injection. Characteristics of the included studies are summarized in Table 1. The sample size ranged from 14 to 96 cases. Assessment results of risk of bias in the randomized trials are shown in Table 2, and the scores of methodological quality of observational studies were all $>5$ scores; thus, overall risk of bias was moderate to good.

\section{Detailed information of TA injection}

The detailed usage of TA is essential for clinical practice. In the included studies, TA was used in two methods: 1) injected into cautery ulcer base after endoscopic surgery (ESD), and it is supposed to prevent the occurrence of stenosis in highrisk patients with size of esophagus defection $>3 / 4$; and 2 ) injected into the narrowest area of the esophagus stenosis combined with ED, and it is supposed to prevent the recurrence of stenosis in esophageal stenosis patients. The TA dose was varied in three studies and fixed in 10 studies ranging from 20 to $100 \mathrm{mg}$, and the dose did not differ significantly in the two injection method studies. More detailed descriptions of TA injection are presented below.

\section{TA injected after surgery (ESD) in five studies}

Hashimoto et al ${ }^{18}$ - Three sessions (post operation day [POD] 3,7 , and 10) of TA $(10 \mathrm{mg} / \mathrm{mL})$ were injected in aliquots of $0.2 \mathrm{~mL}$ ( $2 \mathrm{mg}$ ) evenly ( $1 \mathrm{~cm}$ apart). A 25 -gage, $4 \mathrm{~mm}$ needle, and a $1 \mathrm{~mL}$ syringe were used. The total dose (range 18-62 $\mathrm{mg}$ ) and the number of injections (range 9-31) per session were dependent on the size of resection.

Hanaoka et $\mathrm{al}^{20}$ - A single session of TA $(5 \mathrm{mg} / \mathrm{mL}$, diluted in $0.9 \%$ physiological solution) was injected in $0.5-1.0 \mathrm{~mL}$ evenly. A 25 -gage needle was used. From the margins of the ulcer, linear injections were given from distal to proximal side of the ulcer margin. The total dose was fixed (100 mg) and the number of injections (range 20-40) was dependent on the size of resection.

Mori et $\mathrm{a}^{23}$ - Two sessions (POD 5 and 12) of TA were injected in aliquots of $2 \mathrm{mg}$ evenly. The total dose (40 mg) and the number of injections (20) were both fixed. The concentration and volume were unclear.

Takahashi et $\mathrm{al}^{25}$ - A single session of TA $(10 \mathrm{mg} / \mathrm{mL})$ was injected in aliquots of $0.5 \mathrm{~mL}$ evenly $(1 \mathrm{~cm}$ apart). The total dose and the number of injections were unclear.

Kim et $\mathrm{al}^{26}$ - A single session of TA $(10 \mathrm{mg} / \mathrm{mL})$ was injected in aliquots of $1 \mathrm{~mL}$ evenly $(1 \mathrm{~cm}$ apart). The total dose (range $20-50 \mathrm{mg}$ ) and the number of injections (range 2-5) were dependent on the size of resection.

\section{TA injected combined with ED in eight studies Camargo et $\mathrm{al}^{15}$ - A single session of TA $(10 \mathrm{mg} / \mathrm{mL})$ was injected in aliquots of $1 \mathrm{~mL}$ into four quadrants evenly before dilation. The total dose $(40 \mathrm{mg})$ was fixed.}




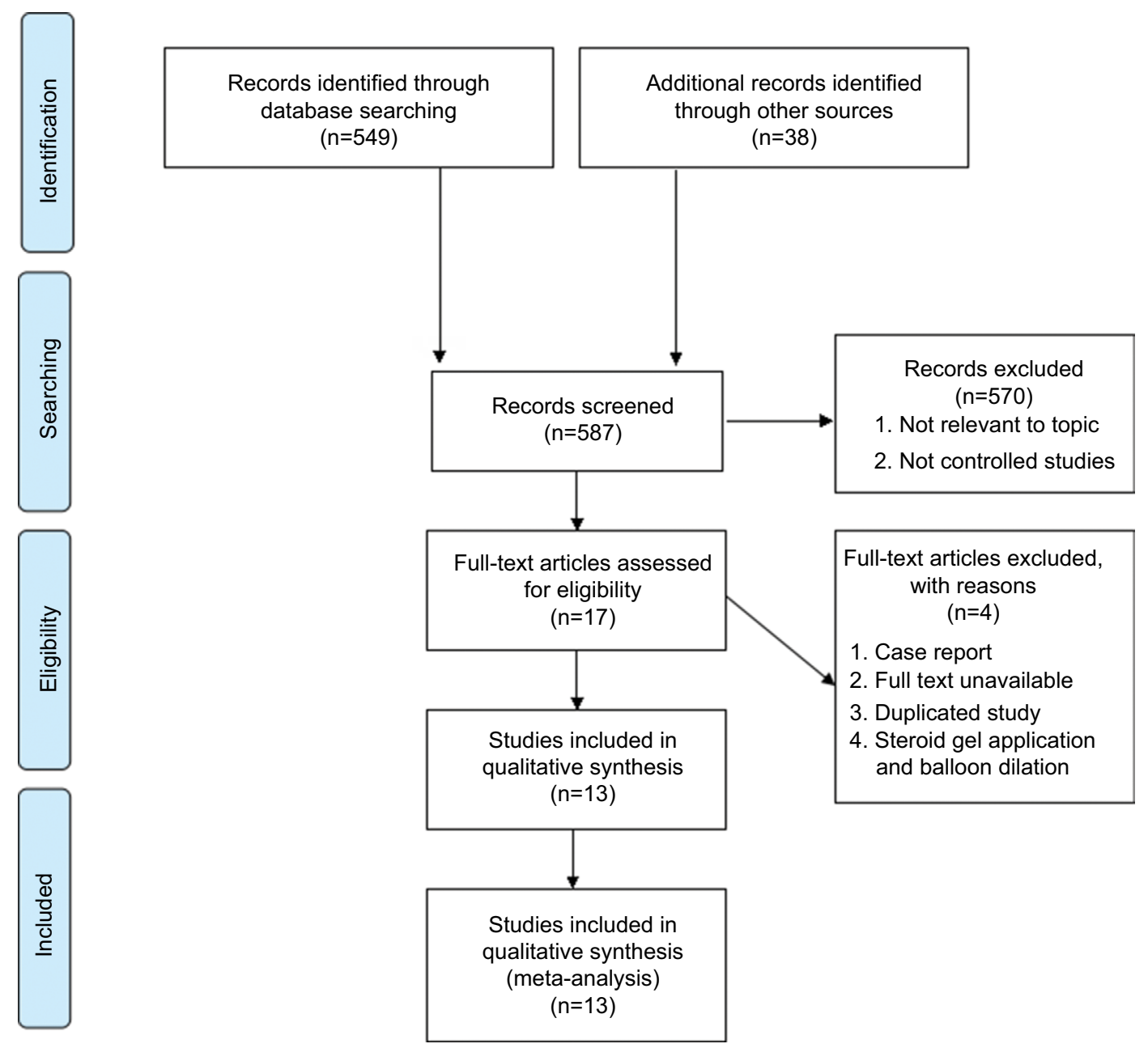

Figure I Flowchart of the study inclusion.

Note: (C) 2009 Moher et al, Moher D, Liberati A, Tetzlaff J, Altman DG; The PRISMA Group (2009) Preferred Reporting Items for Systematic Reviews and Meta-Analyses: The PRISMA Statement. PLoS Med 6(7):el000097. ${ }^{35}$ For more information, visit www.prisma-statement.org.

Altintas et a ${ }^{16}-$ A single session of TA $(8 \mathrm{mg} / \mathrm{mL})$ was injected in aliquots of $1 \mathrm{~mL}$ into four quadrants by a sclerotherapy 21-gage needle evenly before dilation. The total dose (32 mg) was fixed.

Ramage et al ${ }^{17}$ - A single session of TA $(40 \mathrm{mg} / \mathrm{mL})$ was injected in aliquots of $0.5 \mathrm{~mL}$ into four quadrants by a sclerotherapy 22 -gage needle evenly before dilation. The total dose $(80 \mathrm{mg})$ was fixed.

Orive-Calzada et a ${ }^{19}$ - Similar to Ramage et $\mathrm{al}^{17}$, except for a sclerotherapy, 23-gage needle used.

Hirdes et $\mathrm{al}^{21}$ - Same as Orive-Calzada et $\mathrm{al}^{19}$.

Liao et $\mathrm{al}^{22}$ - Two sessions of TA (POD 1 and POD 7) were injected in aliquots of $10 \mathrm{mg}(1 \mathrm{~mL})$ into the borders of the tears and stenosis after dilation. The total dose $(<80$ $\mathrm{mg}$ ) and the number of injections were dependent on the size of stenosis.
Pereira-Lima et $\mathrm{al}^{24}$ - A single session of TA ( $40 \mathrm{mg}$ ) was injected into the borders of the tears after dilation. The concentration, volume, and the number of injections were unclear.

Sugimura et $\mathrm{al}^{27}$ - A single session of TA $(10 \mathrm{mg} / \mathrm{mL})$ was injected in aliquots of $1 \mathrm{~mL}$ into five points around the circle of the anastomosis by a 25 -gage needle evenly after dilation. The total dose $(50 \mathrm{mg})$ was fixed.

\section{Stenosis rate}

The data of stenosis rate were available for 11 studies $(n=423$ patients). In the pooled analysis, TA injection decreased the incidence of stenosis compared with control $(\mathrm{RD}=-0.34$, 95\% CI [-0.54, -0.13$], P<0.01)$; however, significant heterogeneity was detected $\left(P^{2}=90 \%\right)$.

Considering the clinical heterogeneity, subgroup analysis through separating two injection methods was first conducted. 


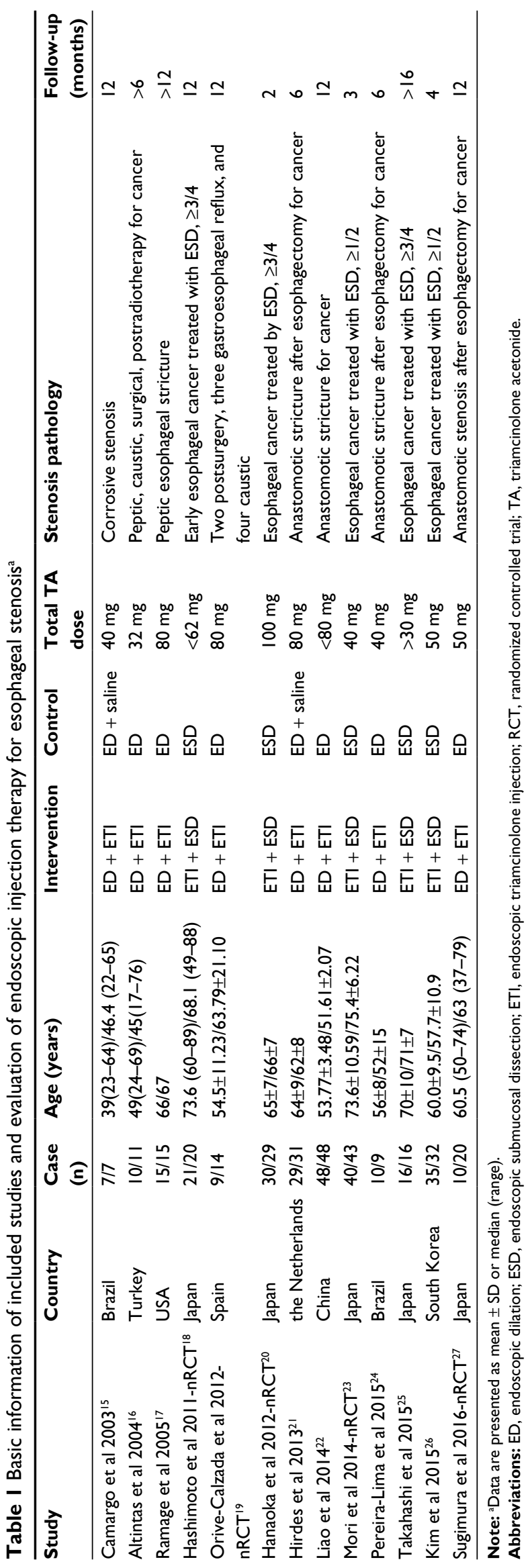

Sensitivity analysis, through a funnel plot, identified that one study that injected TA after ESD for mucosal defect of circumference $<3 / 4$ was responsible for the statistical heterogeneity, ${ }^{23}$ as shown in Figure 2.

After omitting this study, overall heterogeneity was decreased to $71 \%$ and subgroup heterogeneity in the method after endoscopic surgery was decreased from $96 \%$ to $34 \%$. In the new pooled subgroup analysis, TA injection after endoscopic surgery (mucosal defect of circumference $\geq 3 / 4$ ) reduced the risk of occurrence of stenosis $(\mathrm{RD}=-0.51,95 \%$ CI [ $-0.64,-0.39], P<0.01$; Figure 3); meanwhile, TA injection combined with ED reduced the risk of recurrence of stenosis $(\mathrm{RD}=-0.28,95 \% \mathrm{CI}[-0.47,-0.08], P<0.01$; Figure 2$)$.

Because randomized trials represented higher level evidence with better methodological quality than observational studies, meta-analysis of only randomized trials for two different TA injection methods was performed.

In the new pooled analysis, only one randomized trial investigated efficacy of TA injection after endoscopic surgery, ${ }^{25}$ however, the difference was not statistically significant because of insufficient sample size under test powder, as stated by the original authors. Similar to previous evaluations, TA injection combined with ED still significantly reduced the risk of recurrence of stenosis $(\mathrm{RD}=-0.32,95 \% \mathrm{CI}$ [-0.57, -0.07], $P=0.01$; Figure 4).

At the time of TA injection after surgery, two studies performed ETI only once, while the other two studies performed ETI two and three times, respectively. We performed another meta-analysis to assess whether the injection time influenced the results.

In the new pooled analysis, no significant difference was found in subgroups of TA injection performed only one time $(\mathrm{RD}=-0.42,95 \%$ CI $[-0.72,-0.12], P<0.001)$ and more than one time $(\mathrm{RD}=-0.60,95 \% \mathrm{CI}[-0.78,-0.42], P<0.01$; Figure 5).

At the timing of TA injection combined with ED, TA was injected before dilation in five studies, and after dilation in three studies. We performed another meta-analysis to assess whether the sequence influenced the results.

In the new pooled analysis, TA injection before ED significantly reduced the recurrence of stenosis $(\mathrm{RD}=-0.28,95 \%$ $\mathrm{CI}[-0.48,-0.08], P<0.01)$, while no statistical significance was found in subgroup of TA injection after ED reduced the recurrence of stenosis ( $\mathrm{RD}=-0.29,95 \%$ CI $[-0.89,0.31]$, $P=0.34$ ) as shown in Figure 6.

To investigate the relationship between TA dose and incidence of stenosis, a subgroup analysis through separating three kinds of TA dose was then conducted. With high 
Table 2 Risk of bias evaluation based on Jadad scale for randomized studies and Newcastle-Ottawa Quality Assessment Scale for nonrandomized studies

\begin{tabular}{|c|c|c|c|}
\hline Randomized studies & Jadad score & Nonrandomized studies & Newcastle score \\
\hline Camargo et al $2003^{15}$ & 3 & Hashimoto et al $201 \mathrm{I}^{18}$ & 5 \\
\hline Altintas et al $2004^{16}$ & 5 & Orive-Calzada et al $2012^{19}$ & 7 \\
\hline Ramage et al $2005^{17}$ & 4 & Hanaoka et al $2012^{20}$ & 6 \\
\hline Hirdes et al $2013^{21}$ & 6 & Mori et al $2014^{23}$ & 5 \\
\hline Liao et al $2014^{22}$ & 3 & Sugimura et al $2016^{27}$ & 8 \\
\hline Pereira-Lima et al $2015^{24}$ & 5 & & \\
\hline Takahashi et al $2015^{25}$ & 5 & & \\
\hline Kim et al $2015^{26}$ & 6 & & \\
\hline
\end{tabular}

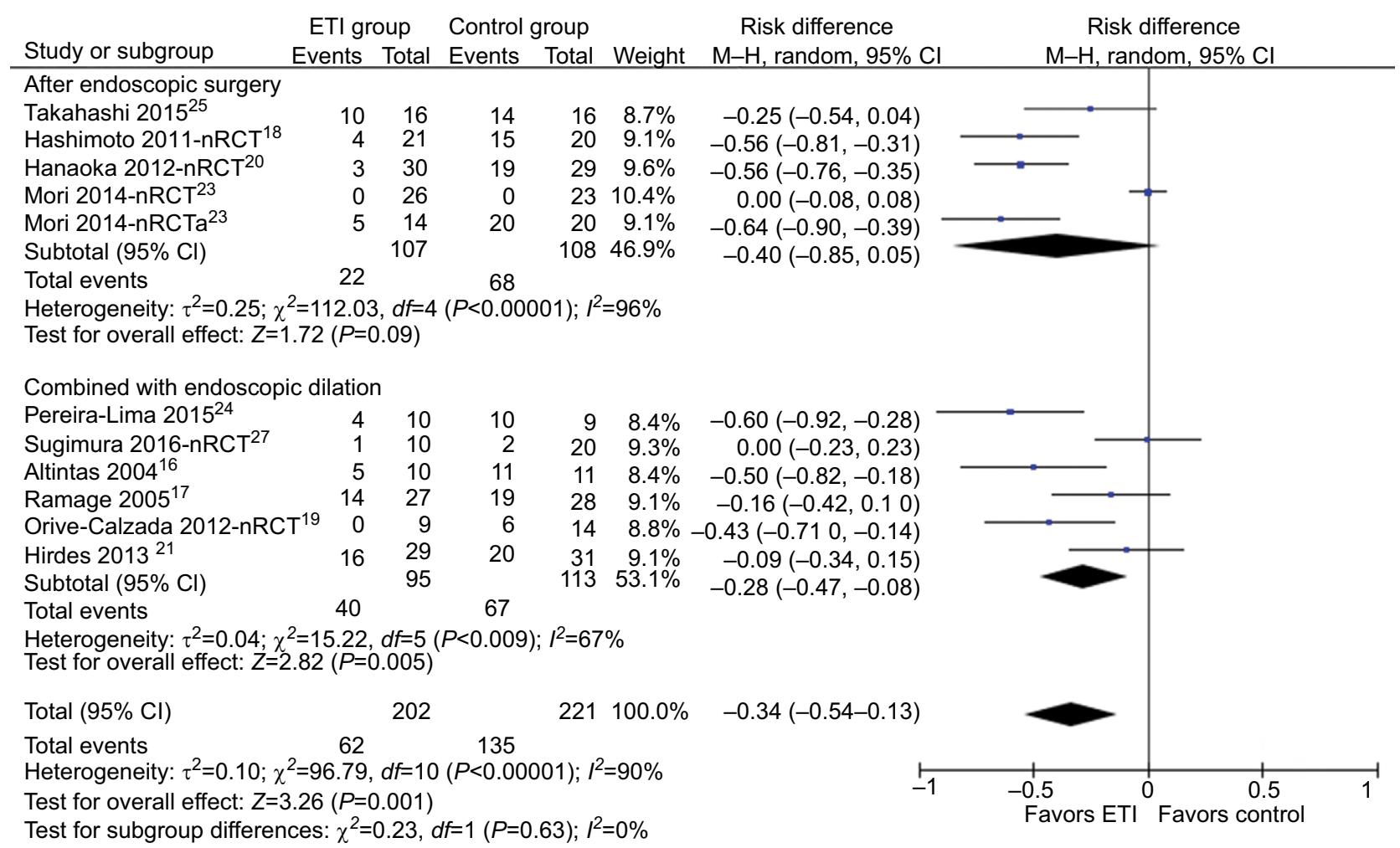

Figure 2 Stenosis incidence of triamcinolone acetonide injection at different timings. Abbreviation: $\mathrm{M}-\mathrm{H}$, Mantel-Haenszel.

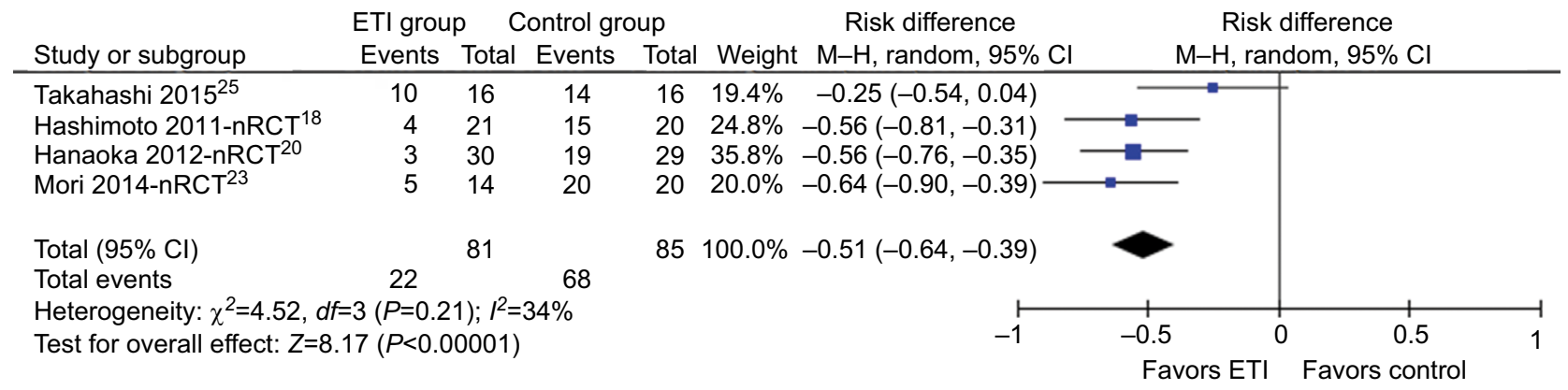

Figure 3 Stenosis incidence of triamcinolone acetonide injection after endoscopic surgery (omitting outlier). Abbreviation: $\mathrm{M}-\mathrm{H}$, Mantel-Haenszel.

level of heterogeneity detected, the results showed that TA injection significantly reduced the incidence of stenosis in $>50 \mathrm{mg}$ subgroup $(\mathrm{RD}=-0.31,95 \%$ CI $[-0.55,-0.08]$,
$P=0.009)$ and specific dose subgroup dependent on the size of stenosis $(\mathrm{RD}=-0.47,95 \% \mathrm{CI}[-0.68,-0.25], P<0.0001)$, while no significantly reduced incidence of stenosis was 


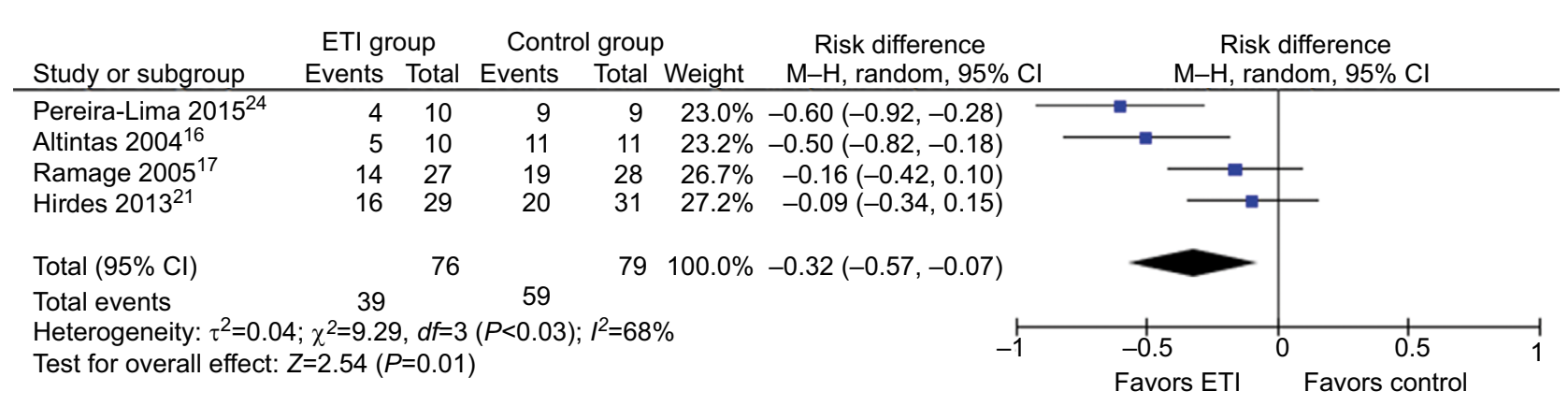

Figure 4 Stenosis incidence of triamcinolone acetonide injection combined with endoscopic dilation in randomized studies.

Abbreviation: M-H, Mantel-Haenszel.

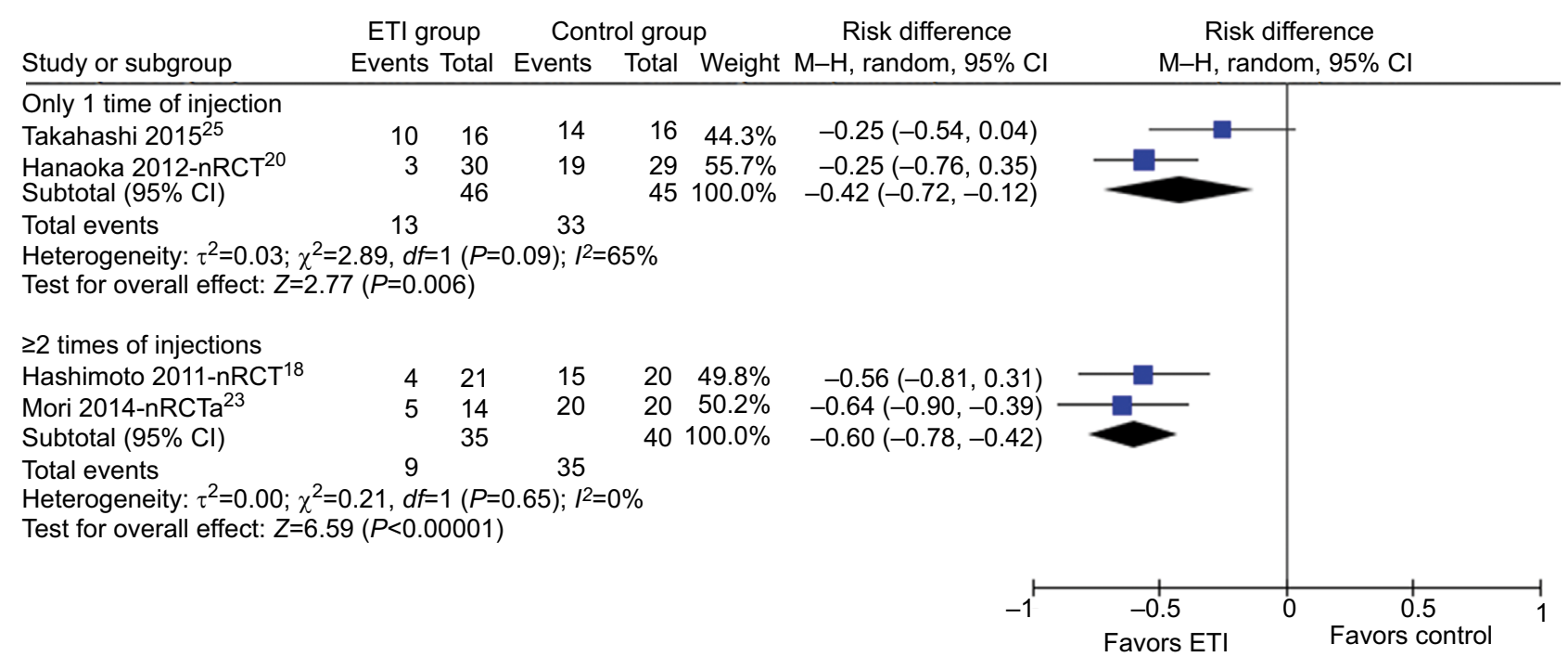

Figure 5 Stenosis incidence of triamcinolone acetonide injection performed different times after surgery.

Abbreviation: M-H, Mantel-Haenszel.

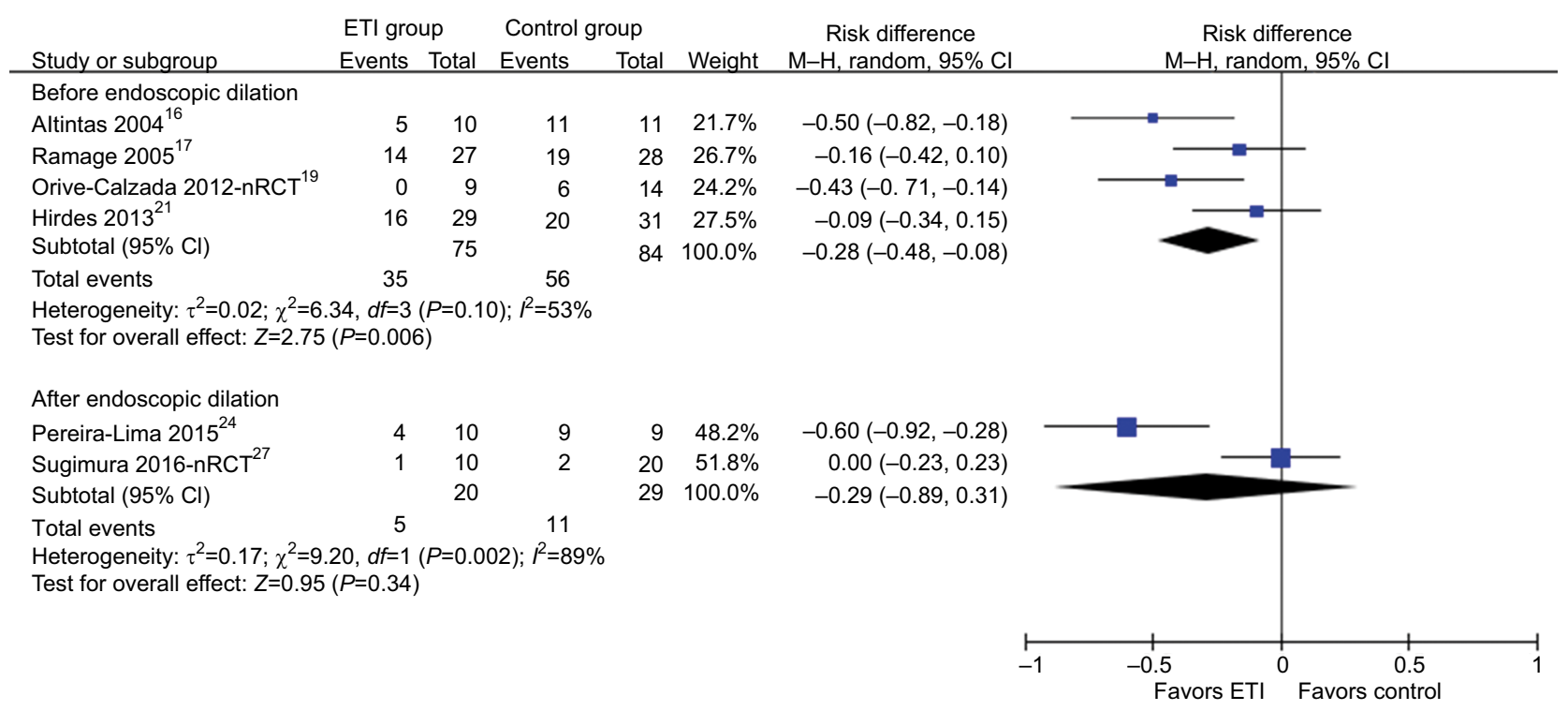

Figure 6 Stenosis incidence of triamcinolone acetonide injection before and after endoscopic dilation.

Abbreviation: M-H, Mantel-Haenszel. 
found in $\leq 50 \mathrm{mg}$ subgroup $(\mathrm{RD}=-0.28,95 \% \mathrm{CI}[-0.71$, $0.15], P=0.21$ ), as shown in Figure 7.

\section{Required ED sessions}

Nine studies reported the number of ED sessions required for resolution of stenosis ( $\mathrm{n}=334)$, and quantitative analysis was available for six studies ( $\mathrm{n}=204)$, as shown in Figure 8. The MD in the number of required sessions was -3.66 in favor of TA injection after endoscopic surgery $(95 \% \mathrm{CI}$ $[-5.87,-1.46], P<0.01)$, and -0.71 in favor of TA injection combined with ED $(95 \% \mathrm{CI}[-1.39,-0.04], P<0.05)$.

The other three studies did not present the data as mean and standard difference, and thus they were not combined and analyzed. ${ }^{18,20,27}$ Required ED sessions were significantly reduced in TA injection compared with control in all the three studies including Hashimoto et al (mean 1.7 [range 0-15] vs 6.6 [0-22]; $P<0.001$ ), Hanaoka et al (median 0 [range 0-2] vs $2[0-15] ; P<0.0001$ ), and Sugimura et al (median 2.5 [range $1-6]$ vs $4.5[1-20] ; P<0.05)$.

\section{Dysphagia-free time}

Regarding the dysphagia-free time in the method of TA injection combined with ED, only three studies reported the data. Statistical heterogeneity across the studies was detected $\left(I^{2}=93 \%\right)$, and sensitivity analysis through a funnel plot found one study to be an outlier. ${ }^{22}$ When this study was excluded, the heterogeneity significantly decreased to $0 \%$. In the new pooled analysis, TA injection also achieved a significant improvement in dysphagia-free time (SMD $=1.66,95 \% \mathrm{CI}$ $[1.15,2.18], P<0.001$; Figure 9).

\section{Complications}

Safety analysis based on the data of complications was available for seven studies ( $\mathrm{n}=380)$ as shown in Table 3 . In the pooled analysis, there were no significant differences in aspects of perforation $(2.80 \%$ vs $3.45 \%)$, bleeding $(1.64 \%$ vs $5.34 \%$ ), infection $(6.49 \%$ vs $3.80 \%)$, and total incidence (5.52\% vs $7.04 \%)$.

For perforation, there were three cases in TA injection group and four cases in control group with ED. In the study of Mori et al, ${ }^{23}$ the only case of perforation in a 78-yearold woman was stated to be mainly caused by TA injection perpendicular to the muscle layers on POD 5 and confirmed on POD 12, and was handled by clip gripper in two times. In the study of Altintas et al, ${ }^{16}$ the cases of perforation were stated to be suffered from caustic stenosis.
The shape of the inverted funnel plot was to some extent symmetrical, indicating low risk of publication bias located in complications (Figure 10).

\section{Discussion}

The diagnosis of esophagus stenosis after surgery was mostly confirmed after failure of passing by an adult gastroscope. Various kinds of therapeutic options have been developed. In the current study, we aimed to evaluate a practical method of using local TA injection in the management of both occurrence and recurrence of esophagus stenosis.

Based on the pooled analysis results, TA injection was safe without increased incidence of complications. In the current analysis, three cases of perforation, two cases of bleeding, and five cases of infection were reported. Among them, perforation was a serious treatment-related complication, and mostly to be handled through invasive method. It was worried that steroid injection appeared to increase the risk of perforation in balloon dilation for preventing strictures; actually statistical analysis in our study and another study using combination therapy of both prophylactic and subsequent TA injection showed no significant difference. ${ }^{27}$ Although steroid-related complication is rare in a relatively low dose of local injection than oral, more attention needs to be paid all the time to patients taking platelet anticoagulants or antiplatelet agents.

Based on this meta-analysis, primary TA injection after surgery for preventing esophageal stenosis was effective. Current analysis mainly included stenosis patients because of injury after esophageal cancer surgery. Although different mechanism might be existed for different kinds of etiology, the efficacy of TA injection for preventing peptic, caustic, and postradiotherapy injuries was not investigated. In clinic, prophylactic TA should be used for surgery patients diagnosed with esophageal cancer and varices adopted ESD/esophagectomy and esophageal transection, according to mucosal defect characteristics such as circumferential extension $(>3 / 4)$, and histological depth (muscle layer damage), longitudinal mucosal defect length $(\geq 5 \mathrm{~cm}) .{ }^{28-30}$ According to the reports in the included studies, TA injection can be performed only one time immediately after surgery or more times during 12 days post operation, and the dose of TA was mainly according to the defect size, which ranged from $18 \mathrm{mg}$ to a maximum of 100 $\mathrm{mg}$ for one person. Detailed information on injection is listed in Results section, and an injection in aliquots of $0.2-0.5 \mathrm{~mL}$ evenly $(0.5-1 \mathrm{~cm}$ apart) seemed to be easily realized. Other things that need to be carefully performed are to avoid injecting into muscle layer, and meanwhile to treat reflux disease 


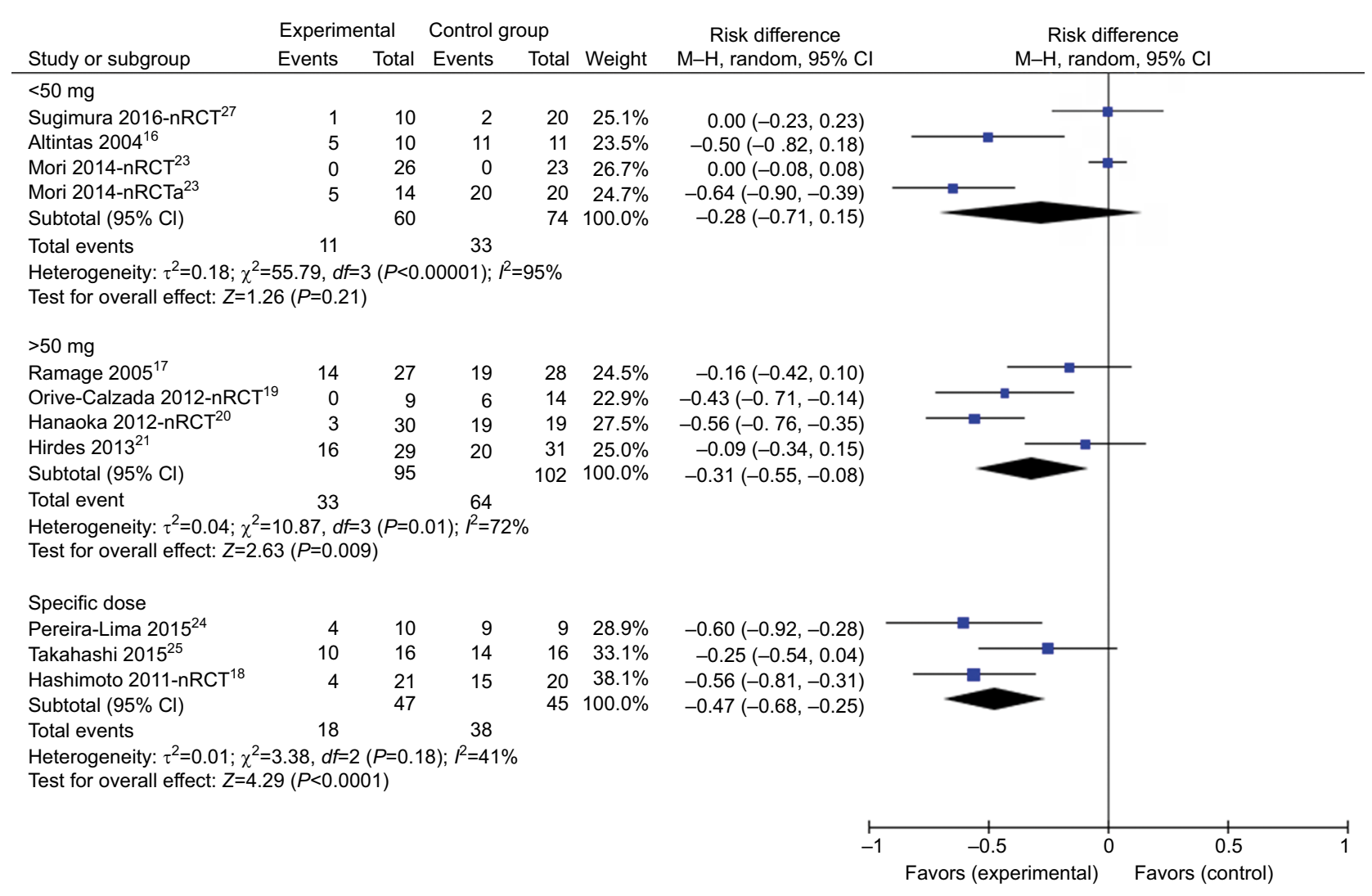

Figure 7 Stenosis incidence of triamcinolone acetonide injection for different doses.

Abbreviation: $\mathrm{M}-\mathrm{H}$, Mantel-Haenszel.

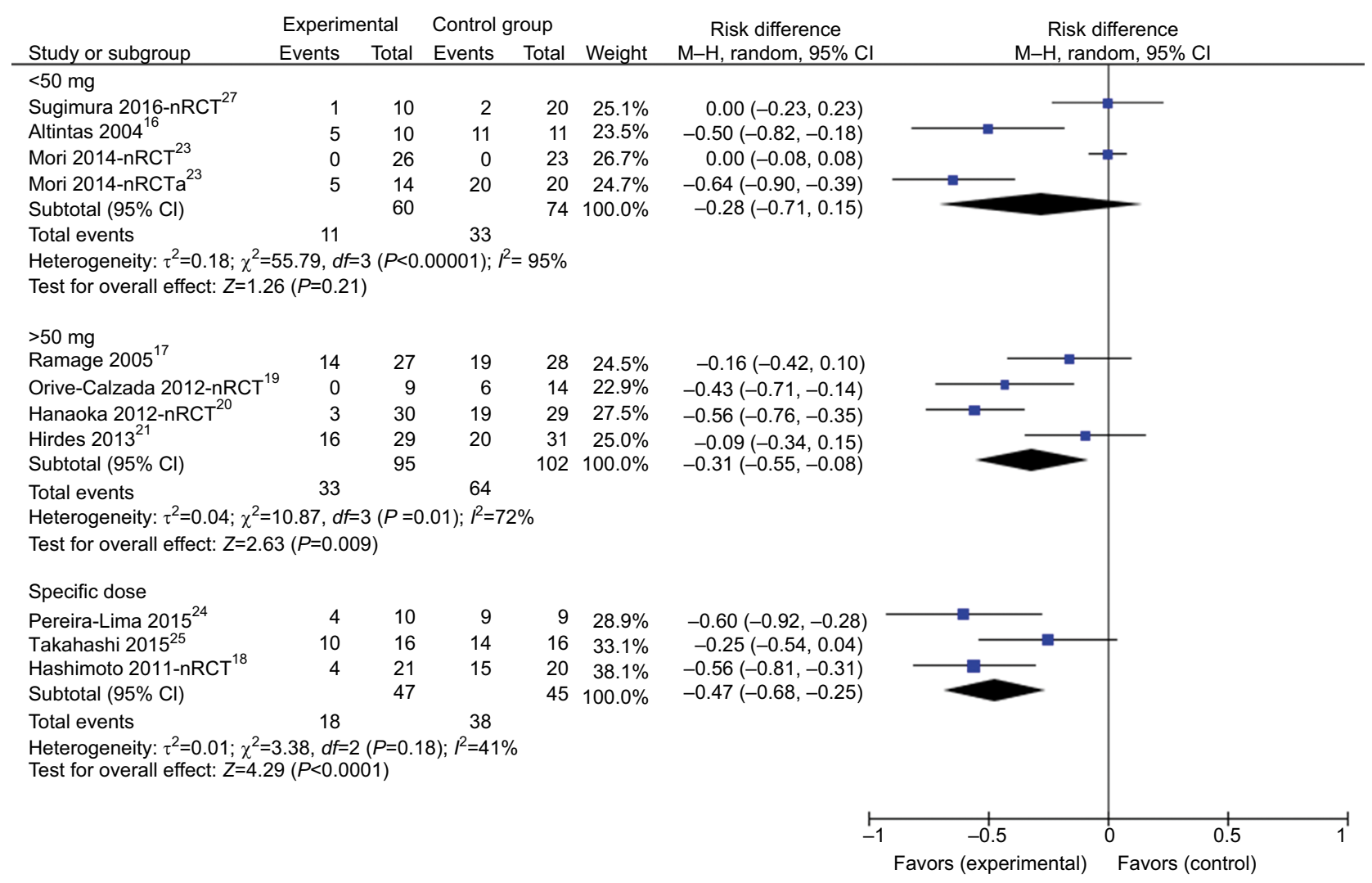

Figure 8 Required ED sessions for resolution of stenosis after triamcinolone acetonide injection at different timings.

Abbreviations: ED, endoscopic dilation; $\mathrm{M}-\mathrm{H}$, Mantel-Haenszel. 


\begin{tabular}{|c|c|c|c|c|c|c|c|}
\hline \multirow[b]{2}{*}{ Study or subgroup } & \multicolumn{3}{|c|}{ ETI group } & \multicolumn{4}{|c|}{ Control group } \\
\hline & Means & SD & Total & Means & s SD & Total & Weight \\
\hline $\begin{array}{l}\text { Itintas } 2004^{16} \\
\text { Hirdes } 2013^{21}\end{array}$ & $\begin{array}{r}24 \\
3.6\end{array}$ & $\begin{array}{r}12.75 \\
1.37\end{array}$ & $\begin{array}{r}10 \\
29\end{array}$ & $\begin{array}{r}5.18 \\
1.4\end{array}$ & $\begin{array}{l}5.06 \\
1.36\end{array}$ & $\begin{array}{l}11 \\
31\end{array}$ & $\begin{array}{l}23.1 \% \\
76.9 \%\end{array}$ \\
\hline Liao $2014^{22}$ & 145.35 & 9.52 & 48 & 100.68 & 12.34 & 48 & $0.0 \%$ \\
\hline $\begin{array}{l}\text { Total }(95 \% \mathrm{Cl}) \\
\text { Heterogeneity: } \chi^{2}= \\
\text { Test for overall effe }\end{array}$ & $\begin{array}{l}0.25, d f= \\
\text { ct: } Z=6.3\end{array}$ & 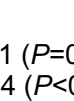 & $\begin{array}{r}39 \\
.62) ;\end{array}$ & $\left.\right|^{2}=0 \%$ & & 42 & $100.0 \%$ \\
\hline
\end{tabular}

SMD SMD

IV, fixed, $95 \% \mathrm{Cl}$

IV, fixed, $95 \% \mathrm{Cl}$

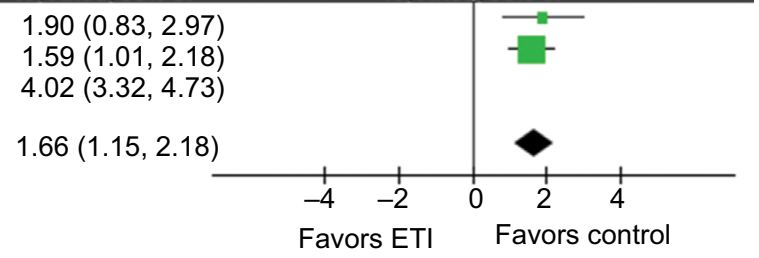

Figure 9 Dysphagia-free time after triamcinolone acetonide injection combined with endoscopic dilation. Abbreviation: SMD, standard mean difference.

Table 3 Complication incidence of triamcinolone acetonide injection for stenosis in fixed-effects model

\begin{tabular}{|c|c|c|c|c|c|c|}
\hline & \multirow[t]{2}{*}{ Studies/references } & \multirow[t]{2}{*}{ Case (n) } & \multicolumn{2}{|l|}{ Incidence (\%) } & \multirow{2}{*}{$\begin{array}{l}\text { Risk difference } \\
{[95 \% \mathrm{Cl}]}\end{array}$} & \multirow[t]{2}{*}{$P$-value } \\
\hline & & & Intervention & Control & & \\
\hline Perforation & $16,19,22,23$ & 223 & $3 / 107$ (2.80\%) & $4 / 116(3.45 \%)$ & $-0.01[-0.06,0.04]$ & 0.82 \\
\hline Bleeding & $21,22,26,27$ & 253 & $2 / 122(1.64 \%)$ & $7 / 131$ (5.34\%) & $-0.04[-0.09,0.01]$ & 0.13 \\
\hline Infection & 21,22 & 156 & $5 / 77$ (6.49\%) & $3 / 79$ (3.80\%) & $0.03[-0.05,0.10]$ & 0.46 \\
\hline Total & $16,19,21-23,26,27$ & 380 & $10 / 181$ (5.52\%) & $14 / 199$ (7.04\%) & $-0.01[-0.04,0.02]$ & 0.53 \\
\hline
\end{tabular}

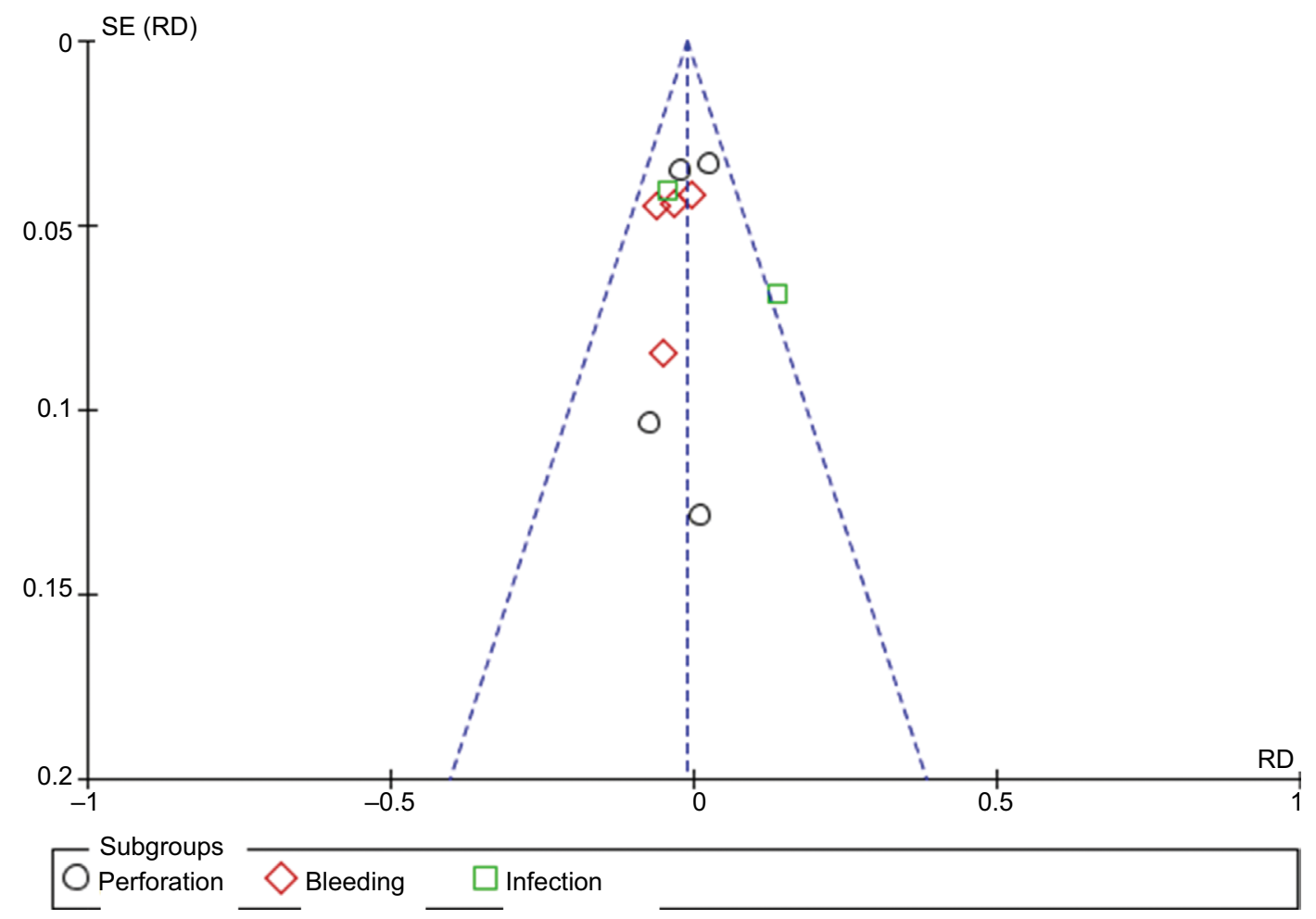

Figure 10 Inverted funnel plot indicating the risk of publication bias located in complications. Abbreviations: SE, standard error; RD, risk difference.

of causative factor, which would be risk factors for incidence of delayed perforation and refractory stenosis.

Currently, esophageal stenosis still occurs even after preventive TA injection, and the reported rate after only one time TA injection was about $17 \%,{ }^{31}$ whereas, no clinical data were found for the stenosis occurrence after two or more times of TA injection. From large-scale retrospective analysis, circumferential extension of mucosal defect $>5 / 6$ and $7 / 8$ increased the likelihood of stenosis occurrence after a single preventive TA injection. ${ }^{31,32}$ Unavoidably, ED was required in the sequence treatment, and current analysis revealed that a combination of ED and TA also showed significant clinical benefits for refractory stenosis (more than three sessions of ED requirement) recurrence.

Therapeutic effect of the combination of ED and TA injection mainly contained a reduced rate of stenosis recurrence 
and also a reduced requirement of ED sessions. While, in the included studies, almost all of them achieved an improvement in required ED sessions other than recurrence rate. To perform the combination therapy, relief of dysphagia symptom in patients is essential, and endoscopic balloon dilation and bougies dilation are both acceptable to reach a diameter of at least $14-16 \mathrm{~mm} .{ }^{21}$ Besides, TA injection for preventing recurrence of stenosis was also revealed to be effective. Detailed information of TA injection procedure is listed in the "Results" section, while a question of relative sequence of ED and TA injection was found to be important and unclear for practice. In the included studies before the year 2014, TA injection was performed before ED, which ensured that injected TA spread into all of the stenosis area; ${ }^{15-17,19,21}$ but in studies after 2014, TA injection was performed after ED, which made TA located only into tears and strictures, and this may lead to different outcomes. ${ }^{22,24,27}$ Based on limited data in our analysis, TA injected before ED seemed to be better in lowering the risk of stenosis recurrence.

\section{Limitations}

The major limitation of this review was the heterogeneity of inclusion of both randomized trials and observational studies in pooled analysis, which might induce selection bias. But, such an inclusion criterion ensured a total of 575 participants, which would be sufficient to achieve the required sample size for statistical analysis. There are also some differences in the included studies: 1) The dose and usage of TA were not completely same, and this might also have influence on the outcomes although the interventions in each trial were comparable. For clinical practice, the specific dose was recommended to be determined for each specific patient based on defect size. 2) Surgeons' experience in conducting the ED may also partly influence the treatment efficacy, and learning curve seemed to be missing in most of the studies. 3) The choice of oral steroid other than local injection is not completely clear, although concerns existed for its high dose; relevant comparison studies were warranted. ${ }^{33,34}$

\section{Conclusion}

TA injection therapy after esophageal cancer surgery and combined with ED are both effective and safe in the management of stenosis, as it reduces the risk of stenosis and sequentially the required ED sessions without increasing complications.

\section{Acknowledgment}

This study was supported by National Key R\&D Program of China (No. 2017YFC0907104).

\section{Disclosure}

The authors report no conflicts of interest in this work.

\section{References}

1. Kim JH, Shin JH, Song HY. Benign strictures of the esophagus and gastric outlet: interventional management. Korean J Radiol. 2010;11(5):497-506.

2. Isomoto H, Yamaguchi N, Nakayama T, et al. Management of esophageal stricture after complete circular endoscopic submucosal dissection for superficial esophageal squamous cell carcinoma. BMC Gastroenterol. 2011;11(1):46.

3. Isomoto H, Shikuwa S, Yamaguchi N, et al. Endoscopic submucosal dissection for early gastric cancer: a large-scale feasibility study. Gut. 2009;58(3):331-336.

4. Ohki T, Yamato M, Ota M, et al. Prevention of esophageal stricture after endoscopic submucosal dissection using tissue-engineered cell sheets. Gastroenterology. 2012;143(3):582-588.e2.

5. Ono S, Fujishiro M, Niimi K, et al. Long-term outcomes of endoscopic submucosal dissection for superficial esophageal squamous cell neoplasms. Gastrointest Endosc. 2009;70(5):860-866.

6. van Heijl M, Gooszen JA, Fockens P, et al. Risk factors for development of benign cervical strictures after esophagectomy. Ann Surg. 2010;251(6):1064-1069.

7. van Boeckel PG, Siersema PD. Refractory esophageal strictures: what to do when dilation fails. Curr Treat Options Gastroenterol. 2015;13(1):47-58

8. de Moura EGH, Orso IRB, Aurélio EF, de Moura ETH, de Moura DTH, Santo MA. Factors associated with complications or failure of endoscopic balloon dilation of anastomotic stricture secondary to Roux-en-Y gastric bypass surgery. Surg Obes Relat Dis. 2016;12(3):582-586.

9. Holder TM, Ashcraft KW, Leape L. The treatment of patients with esophageal strictures by local steroid injections. J Pediatr Surg. 1969;4(6):646-653.

10. Divarci E, Celtik U, Dokumcu Z, Ozcan C, Erdener A. The efficacy of intralesional steroid injection in the treatment of corrosive esophageal strictures in children. Surg Laparosc Endosc Percutan Tech. 2016;26(6):e122-e125.

11. Ahuja RB, Chatterjee P. Comparative efficacy of intralesional verapamil hydrochloride and triamcinolone acetonide in hypertrophic scars and keloids. Burns. 2014;40(4):583-588.

12. Higgins JPT, Green S, editors. Cochrane Handbook for Systematic Reviews of Interventions Version 5.1.0 [updated March 2011]. The Cochrane Collaboration, 2011. Available from: https://training. cochrane.org/handbook. Accessed September 21, 2018.

13. Moher D, Shamseer L, Clarke M, et al. Preferred reporting items for systematic review and meta-analysis protocols (PRISMA-P) 2015 statement. Syst Rev. 2015;4(1):1.

14. Margulis AV, Pladevall M, Riera-Guardia N, et al. Quality assessment of observational studies in a drug-safety systematic review, comparison of two tools: the Newcastle-Ottawa Scale and the RTI item bank. Clin Epidemiol. 2014;6:359-368.

15. Camargo MA, Lopes LR, Grangeia TAG. USO de corticosteróides após Dilatação esofágica EM pacientes com estenose corrosivos: prospective, Estudo randomizado e DUPLO-Cego [Use of corticosteroids after esophageal dilations on patients with corrosive stenosis: prospective, randomized and double-blind study]. Rev Assoc Med Bras (1992). 2003;49 (3):286-292. Português.

16. Altintas E, Kacar S, Tunc B, et al. Intralesional steroid injection in benign esophageal strictures resistant to bougie dilation. J Gastroenterol Hepatol. 2004;19(12):1388-1391.

17. Ramage JI, Rumalla A, Baron TH, et al. A prospective, randomized, double-blind, placebo-controlled trial of endoscopic steroid injection therapy for recalcitrant esophageal peptic strictures. Am J Gastroenterol. 2005;100(11):2419-2425.

18. Hashimoto S, Kobayashi M, Takeuchi M, Sato Y, Narisawa R, Aoyagi Y. The efficacy of endoscopic triamcinolone injection for the prevention of esophageal stricture after endoscopic submucosal dissection. Gastrointest Endosc. 2011;74(6):1389-1393. 
19. Orive-Calzada A, Bernal-MartinezA, Navajas-Laboa M, et al. Efficacy of intralesional corticosteroid injection in endoscopic treatment of esophageal strictures. Surg Laparosc Endosc Percutan Tech. 2012;22(6):518-522.

20. Hanaoka N, Ishihara R, Takeuchi Y, et al. Intralesional steroid injection to prevent stricture after endoscopic submucosal dissection for esophageal cancer: a controlled prospective study. Endoscopy. 2012;44(11): 1007-1011.

21. Hirdes MM, van Hooft JE, Koornstra JJ, et al. Endoscopic corticosteroid injections do not reduce dysphagia after endoscopic dilation therapy in patients with benign esophagogastric anastomotic strictures. Clin Gastroenterol Hepatol. 2013;11(7):795-801.e1.

22. Liao WQ, Liu XY, Zhang L. Efficacy of combined endoscope and triamcinolone for esophagus benign strictures. China J Endocrinol. 2014;20(10):1102-1105.

23. Mori H, Kobara H, Rafiq K. Local steroid injection prevent deformity and stenosis of stomach after large endoscopic submucosal dissection. Am J Digest Dis. 2014;1(2):136-142.

24. Pereira-Lima JC, Lemos Bonotto M, Hahn GD, et al. A prospective randomized trial of intralesional triamcinolone injections after endoscopic dilation for complex esophagogastric anastomotic strictures: steroid injection after endoscopic dilation. Surg Endosc. 2015;29(5):1156-1160.

25. Takahashi H, Arimura Y, Okahara S, et al. A randomized controlled trial of endoscopic steroid injection for prophylaxis of esophageal stenoses after extensive endoscopic submucosal dissection. BMC Gastroenterol. 2015;5(11):1.

26. Kim B, Lee H, Chung H, et al. The efficacy of topical bupivacaine and triamcinolone acetonide injection in the relief of pain after endoscopic submucosal dissection for gastric neoplasia: a randomized double-blind, placebo-controlled trial. Surg Endosc. 2015;29(3):714-722.

27. Sugimura K, Motoori M, Yano M, et al. Endoscopic steroid injection reduced frequency of repeat dilation in patients with anastomotic stenosis after esophagectomy. Esophagus. 2016;13(1):62-67.
28. Tsujii Y, Hayashi Y, Kawai N, et al. Risk of perforation in balloon dilation associated with steroid injection for preventing esophageal stricture after endoscopic submucosal dissection. Endosc Int Open. 2017;5(7):E573-E579.

29. Miwata T, Oka S, Tanaka S, et al. Risk factors for esophageal stenosis after entire circumferential endoscopic submucosal dissection for superficial esophageal squamous cell carcinoma. Surg Endosc. 2016;30(9):4049-4056.

30. Shi Q, Ju H, Yao LQ, et al. Risk factors for postoperative stricture after endoscopic submucosal dissection for superficial esophageal carcinoma. Endoscopy. 2014;46(8):640-644.

31. NagamiY, Ominami M, Shiba M, et al. Prediction of esophageal stricture in patients given locoregional triamcinolone injections immediately after endoscopic submucosal dissection. Dig Endosc. 2018;30(2): 198-205.

32. Okamoto K, Matsui S, Watanabe T, et al. Clinical analysis of esophageal stricture in patients treated with intralesional triamcinolone injection after endoscopic submucosal dissection for superficial esophageal cancer. Oncology. 2017;93 Suppl 1:9-14.

33. Mizutani T, Tanaka M, Eba J, et al. A Phase III study of oral steroid administration versus local steroid injection therapy for the prevention of esophageal stricture after endoscopic submucosal dissection (JCOG1217, Steroid EESD P3). Jpn J Clin Oncol. 2015;45(11):1087-1090.

34. Kataoka K, Aoyama I, Mizusawa J, et al. A randomized controlled Phase II/III study comparing endoscopic balloon dilation combined with steroid injection versus radial incision and cutting combined with steroid injection for refractory anastomotic stricture after esophagectomy: Japan Clinical Oncology Group Study JCOG1207. Jpn J Clin Oncol. 2015;45(4):385-389.

35. Moher D, Liberati A, Tetzlaff J, Altman DG, The PRISMA Group (2009) Preferred Reporting Items for Systematic Reviews and Meta-Analyses: The PRISMA Statement. PLoS Med 6(7): e1000097.
Cancer Management and Research

\section{Publish your work in this journal}

Cancer Management and Research is an international, peer-reviewed open access journal focusing on cancer research and the optimal use of preventative and integrated treatment interventions to achieve improved outcomes, enhanced survival and quality of life for the cancer patient. The manuscript management system is completely online and includes

\section{Dovepress}

a very quick and fair peer-review system, which is all easy to use. Visit http://www.dovepress.com/testimonials.php to read real quotes from published authors. 